\title{
Magnetic coupling in epitaxial TM/MgO/Fe(001) (TM=FeCo, Fe/Co, Fe) macroscopic and microscopic trilayers
}

\author{
C. Martínez Boubetaa) \\ Instituto de Microelectrónica de Madrid-IMM (CNM-CSIC), Isaac Newton 8-PTM, 28760 Tres Cantos, \\ Madrid, Spain \\ J. M. de Teresa \\ Facultad de Ciencias, Instituto de Ciencia de Materiales de Aragón, Universidad de Zaragoza-CSIC, \\ 50009 Zaragoza, Spain \\ J. L. Costa-Krämer and J. Anguita \\ Instituto de Microelectrónica de Madrid-IMM (CNM-CSIC), Isaac Newton 8-PTM, 28760 Tres Cantos, \\ Madrid, Spain \\ D. Serrate, J. I. Arnaudas, and M. R. Ibarra \\ Facultad de Ciencias, Instituto de Ciencia de Materiales de Aragón, Universidad de Zaragoza-CSIC, \\ 50009 Zaragoza, Spain
}

\author{
A. Cebollada and F. Briones \\ Instituto de Microelectrónica de Madrid-IMM (CNM-CSIC), Isaac Newton 8-PTM, 28760 Tres Cantos, \\ Madrid, Spain
}

(Received 31 October 2002; accepted 11 June 2003)

\begin{abstract}
Multilayered TM/MgO/Fe (001) heterostructures (TM: $\mathrm{FeCo}, \mathrm{Co} / \mathrm{Fe}$, and $\mathrm{Fe}$ ) are grown epitaxially, to study the dependence of the magnetic coupling between the two ferromagnetic electrodes on the insulating $\mathrm{MgO}$ barrier width and the lateral dimensions of the structures. The crystal quality is investigated by reflection high-energy electron diffraction in situ at different growth stages of the $\mathrm{TM} / \mathrm{MgO} / \mathrm{Fe}(001)$ heterostructures. Magnetic characterization by superconducting quantum interference device magnetometry (macroscopic structures) and transverse Kerr effect (microscopic structures) shows clearly independent switching of top and bottom electrodes at large (above $20 \AA$ ) spacer thicknesses for macroscopic films. This independent switching is also observed on patterned structures. For very thin barriers, decreasing the size of the elements in patterned arrays decreases the number of junctions coupled via pinholes. (C) 2003 American Institute of Physics.
\end{abstract}

[DOI: $10.1063 / 1.1598280]$

\section{INTRODUCTION}

The fabrication of metal/insulator magnetic heterostructures has special relevance in the field of magnetic tunnel junctions (MTJ). These structures exhibit a field dependent tunnel—or junction—magnetoresistance (TMR), ${ }^{1}$ which has applications such as magnetic random access memories, programable logic, field sensors, etc. ${ }^{2}$ In the MTJ research field, most of the fabricated structures, and especially those built with transition-metal electrodes, are made of polycrystalline ferromagnets separated by amorphous insulating barriers. These polycrystalline/amorphous systems are of great interest because of their performance and direct technological relevance to industry. On the other hand, fully epitaxial (transition metal/insulator) systems exhibiting TMR allow a direct comparison with state-of-the-art theoretical calculations, where $\mathrm{Fe} / \mathrm{MgO} / \mathrm{Fe}$ is among the most modeled systems. ${ }^{3}$

One of the crucial aspects in the performance of a MTJ is the presence of pinholes in the insulating barrier, revealed to be very important in the transport behavior of tunnel junctions, but also in the magnetization reversal of multilayered heterostructures. In addition, pinholes can affect the indepen-

${ }^{\text {a)} E l e c t r o n i c ~ m a i l: ~ b o u b e t a @ i m m . c n m . c s i c . e s ~}$ dent character of the magnetization reversal of both electrodes by direct ferromagnetic exchange coupling. This last effect on the hysteresis loops has been modeled for antiferromagnetically coupled multilayers, ${ }^{4}$ giving rise to ferromagnetic, antiferromagnetic, or to a mixture of both states depending on pinhole size and separation. However, this characteristic is less studied, and neither the size of the pinholes nor their number or density needed to exchange couple the two electrodes are known.

In this sense, Keavney et al. ${ }^{5}$ studied magnetic coupling in $\mathrm{Fe} / \mathrm{MgO} / \mathrm{Fe}$ epitaxial heterostructures; $\mathrm{Fe}$ ferromagnetic electrodes were coupled for $\mathrm{MgO}$ thicknesses below $75 \AA$, and via transport measurements, no $\mathrm{MgO}$ spacers with thicknesses below $40 \AA$ were found to be insulating. The commented thickness dependence of the switching field was then assigned to a thickness dependence pinhole size or pinhole density, as was observed by scanning electron microscopy.

Interlayer magnetic coupling through $\mathrm{MgO}$ spacers has also been studied by van der Heijden $e t$ al. ${ }^{6}$ who fabricated $\mathrm{Fe}_{3} \mathrm{O}_{4} / \mathrm{MgO} / \mathrm{Fe}_{3} \mathrm{O}_{4}$ trilayers, again finding ferromagnetic coupling due to pinholes for $\mathrm{MgO}$ thicknesses below $13 \AA$ and due to "orange peel" coupling ${ }^{7}$ effects above this thickness. Obviously, a tight control on the spacer quality, with 
absence of pinholes is a big challenge, and was not until very recently that Popova et al. have shown their capability to obtain continuous insulating $\mathrm{MgO}$ spacers as thin as $8 \AA$ in $\mathrm{Fe} / \mathrm{MgO} / \mathrm{Fe}$ epitaxial heterostructures, ${ }^{8}$ and the observation of interlayer antiferromagnetic coupling mediated by spin polarized tunnel electrons between the two Fe electrodes through $\mathrm{MgO}$ layers as thin as $5 \AA^{9}{ }^{9}$

That work claims agreement with theoretical predictions of antiferromagnetic exchange coupling between ferromagnetic electrodes separated by ultrathin insulating spacers. ${ }^{10,11}$ Finally, in the case of structures of lateral dimensions of the order of micrometers, dipolar interactions between top and bottom electrodes are enhanced. This renders at zero field an antiparallel electrode alignment that minimizes the magnetostatic energy of the system, as demonstrated in the specific case of tiled $\mathrm{Fe} / \mathrm{MgO} / \mathrm{Fe}(001)$ trilayered structures. ${ }^{12}$

From the basic point of view, MTJs with identical electrodes simplify the physical interpretation of the results. However, to observe TMR, an antiparallel orientation of the electrode magnetizations at selected field values is mandatory, and thus the two electrodes must have different coercive fields. A different switching field can be achieved by (i) slightly varying the composition of the electrodes, (ii) deposition of additional layers that antiferromagnetically pinexchange bias - the magnetization of one electrode, and (iii) coupling one electrode to additional ferromagnetic layers. As an example of the first approach a study of the magnetization reversal in $\mathrm{Fe}_{0.5} \mathrm{Co}_{0.5} / \mathrm{MgO} / \mathrm{Fe}(001)$ systems is described later. The third approach, also reported in this work, involves making the top Fe layer magnetically harder by depositing Co on top, which grows epitaxially as well. The two ferromagnetic layers are exchange coupled and the magnetization reversal of the combined electrode occurs cooperatively.

Within this scope, this work presents results on the epitaxy and magnetization behavior of metal/insulator magnetic heterostructures. The spacer is $\mathrm{MgO}$ and the electrodes are $\mathrm{Fe}, \mathrm{FeCo}$, and $\mathrm{Fe} / \mathrm{Co}$, which have been the electrodes used in epitaxial magnetic tunnel junctions with $\mathrm{MgO}(001)$ spacers and demonstrated TMR. ${ }^{13,14}$ In addition, magnetization results on structures of both macroscopic and microscopic lateral dimensions are shown.

\section{EXPERIMENT}

The structures are fabricated in an UHV deposition system by the combined use of sputtering and laser ablation, as described elsewhere, ${ }^{15}$ both on $\mathrm{GaAs}(001)$ or $\mathrm{MgO}(001)$ substrates. In both cases, a $100 \AA \mathrm{MgO}$ buffer layer is grown at $450{ }^{\circ} \mathrm{C}$ by normal incidence pulsed laser deposition from a monocrystalline $\mathrm{MgO}$ target in the pressure range of 7 $\times 10^{-9}$ mbar. Deposition rates around $0.17 \AA / \mathrm{s}$ were calibrated ex situ by x-ray low angle reflectometry, profilometer and transmission electron microscopy (TEM) measurements. The role of this $\mathrm{MgO}$ buffer layer onto $\mathrm{GaAs}(001)$ is twofold: it offers an appropriate symmetry and lattice match for the epitaxy of bcc $\mathrm{Fe}(001)$ layers, and it acts as an interdiffusion barrier to avoid incorporation of As from the substrate into the subsequent layers, showing excellent properties as diffusion barrier with thermal stability up to $800^{\circ} \mathrm{C}$ and sig- nificant good electrical insulation characteristics in layered systems. ${ }^{16}$ In addition, this homoepitaxial $\mathrm{MgO}$ buffer layer onto $\mathrm{MgO}(001)$ substrates flattens the original $\mathrm{MgO}$ surface, eliminating all defects and microscopic roughness created by exposure to atmospheric water vapor and the formation of brucite $\mathrm{MgO}(\mathrm{OH})_{2} \cdot{ }^{17}$

On this $\mathrm{MgO}$ buffer layer, $\mathrm{Fe}, \mathrm{Co}$, and $\mathrm{FeCo}$ layers are deposited from individual $\mathrm{Fe}$ and $\mathrm{Co}$ targets. This is performed by triode sputtering at $4 \times 10^{-4}$ mbar Ar pressure with deposition rates around $0.1-0.3 \AA / \mathrm{s}$. $\mathrm{MgO}$ spacer layers were deposited at $7 \times 10^{-9}$ mbar by laser ablation and at $400^{\circ} \mathrm{C}$ substrate temperature. Auger electron spectroscopy measurements confirm the 1:1 composition for the $\mathrm{MgO}$ buffer layers and spacers ${ }^{18}$ as well as the absence of Fe segregation in the $\mathrm{MgO}$ and vice versa. This last point is corroborated by TEM measurements. ${ }^{15}$ The crystalline structure is analyzed by reflection high-energy electron diffraction (RHEED) in situ. The surface morphology at intermediate growth stages and for the final structure is also examined by atomic force microscopy (AFM), scanning areas of up to 5 $\times 5 \mu \mathrm{m}^{2}$. Magnetic properties are studied in a commercial superconducting quantum interference device (SQUID) magnetometer and by transverse magneto-optical Kerr effect (MOKE). Finally, microfabrication techniques [photo and electron (e)-beam lithography, plus $\mathrm{Ar}^{+}$ion milling] are used to define structures in the microscopic range.

\section{RESULTS}

The epitaxial growth of the different layers that form the entire structure is studied in detail by RHEED in situ. The growth of individual $\mathrm{Fe}, \mathrm{Co}$, and $\mathrm{Fe}_{0.5} \mathrm{Co}_{0.5}$ single layers is performed to check their epitaxy previous to the deposition of the multilayered structures. In Fig. 1(b) the RHEED patterns for a $200 \AA \mathrm{Fe}_{0.5} \mathrm{Co}_{0.5}$ film grown at RT are shown. The RHEED patterns for the $\mathrm{MgO}$ buffer layer [Fig. 1(a)] are also presented, and for the sake of comparison we also present in Fig. 1(c) the RHEED patterns for an Fe film grown at RT and subsequently annealed at $400^{\circ} \mathrm{C}$. All patterns are recorded with the incident beam along the same crystallographic directions. Notice sharp and intense diffraction streaks with a well defined spacing that depends on the in-plane crystalline direction. This demonstrates the epitaxial nature of the film. The similarity of both $\mathrm{Fe}$ and $\mathrm{Fe}_{0.5} \mathrm{Co}_{0.5}$ RHEED patterns, added to the presence of $50 \%$ of Fe content in the alloy, allows the identification of a bcc crystalline structure for the $\mathrm{Fe}_{0.5} \mathrm{Co}_{0.5}$ layer. This bcc phase is consistent with the bulk $\mathrm{Fe}-\mathrm{Co}$ phase diagram. ${ }^{19}$ On the other hand, the lattice constant for $\mathrm{Fe}_{0.5} \mathrm{Co}_{0.5}$ is about $2.856 \AA$, ${ }^{20}$ which is close to the $2.866 \AA$ value for bcc Fe. Surface faceting, often observed in $\mathrm{Fe}$ grown on $\mathrm{MgO}$ at $\mathrm{RT},{ }^{15}$ is not present here and even faint Kikuchi lines can be observed for the FeCo pattern aligned along the [100] direction. This confirms the high crystalline quality and improved surface flatness of our FeCo as-grown film compared to the RT Fe epitaxy on $\mathrm{MgO}$. This is probably due not only to different interface energies between $\mathrm{FeCo} / \mathrm{MgO}$ and $\mathrm{Fe} / \mathrm{MgO}$, but also to different Schwoebel barriers distribution for surface diffusion than the ones observed for $\mathrm{Fe} / \mathrm{Fe}$ homoepitaxy. ${ }^{21}$ 

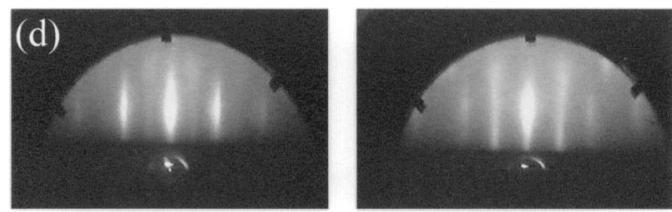

bec Co [110]

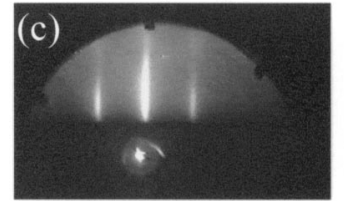

bcc Co [100]

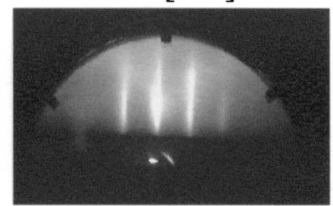

$\mathrm{Fe}[110]$

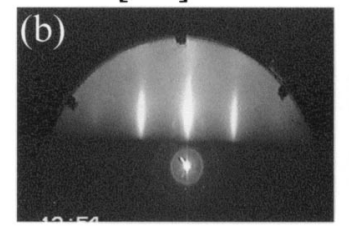

$\mathrm{Fe}[100]$
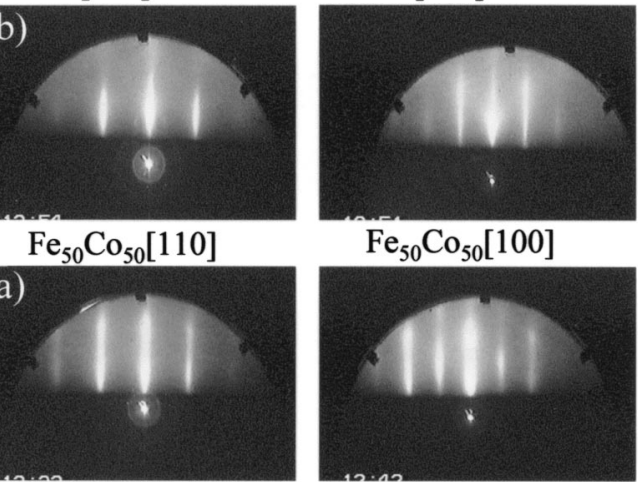

$\mathrm{MgO}[100]$
$\mathrm{Fe}_{50} \mathrm{Co}_{50}[100]$

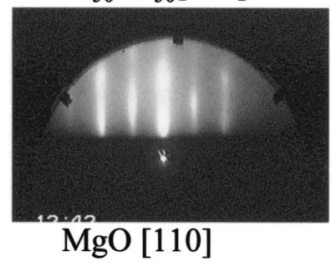

FIG. 1. (a), (b) RHEED patterns showing the epitaxy of $\mathrm{FeCo}(001)$ on $\mathrm{MgO}(001)$. (c) The pattern of a $\mathrm{Fe}(001)$ film on $\mathrm{MgO}(001)$ is also shown to demonstrate the equivalent symmetry. (d) RHEED pattern showing the epitaxy of $\mathrm{Co}$ on $\mathrm{Fe}(001)$.

On the other hand, the RHEED patterns for the $100 \AA$ Co film grown on $\mathrm{Fe}$ (001) are also shown in Fig. 1(d), the same observed symmetry and spacing with respect to $\mathrm{Fe}$ pointing to a bcc Co epitaxial growth at RT. This is consistent with results of previous works ${ }^{22-24}$ where it has been demonstrated that ultrathin Co films grown on $\mathrm{Fe}(001)$ up to 35 ML display a metastable body-centered-tetragonal structure, the equilibrium hcp phase developing at larger Co thickness.

Figure 2 shows a sequence of RHEED patterns for the individual layers of a complete $100 \AA \mathrm{Co} / 100 \AA \mathrm{Fe} / 20 \AA$ $\mathrm{MgO} / 400 \AA \mathrm{Fe}(001)$ epitaxial heterostructure. From bottom to top, first the pattern of the Fe bottom electrode is shown in Fig. 2(a). Deposition of $\mathrm{Fe}$ at RT gives rise to a faceted surface, as evidenced by the chevron like features. This faceting disappears after annealing the $\mathrm{Fe}$ surface at $400{ }^{\circ} \mathrm{C}$, which is going to be the deposition temperature of the next $\mathrm{MgO}$ layer. As shown in Fig. 2(b), the Fe RHEED pattern after annealing exhibits sharp and intense streaks with no indication of faceting. Deposition of $\mathrm{MgO}$ on this flat Fe film yields sharp and intense RHEED diffraction streaks, as shown in Fig. 2(c), confirming the epitaxial $\mathrm{Fe}(001)[110] \| \mathrm{MgO}(001)[100]$ relationship. Finally, in Figs. 2(d) and 2(e) the diffraction patterns corresponding to the successive deposition of $\mathrm{Fe}$ and $\mathrm{Co}$ at RT are shown. The first Fe film is faceted again, and the Co layer deposited on top is somehow more defective as compared to the pattern shown in Fig. 1(b), probably due to the presence of hcp grains. The deposition of the upper electrode-and capping

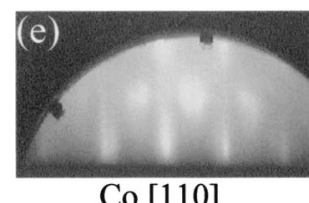

Co [110]

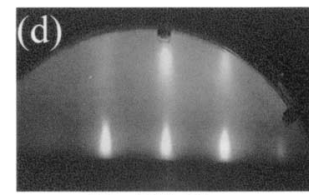

$\mathrm{Fe}[110]$
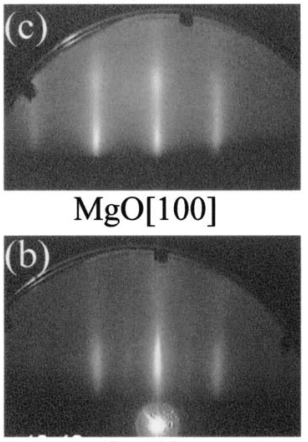

$\mathrm{Fe}[110]$

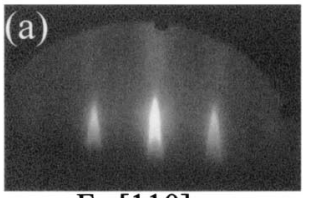

$\mathrm{Fe}[110]$

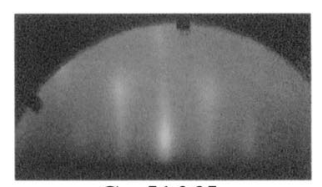

Co $[100]$

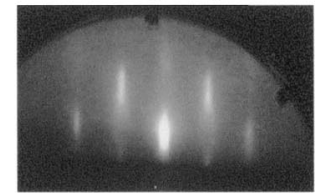

$\mathrm{Fe}[100]$

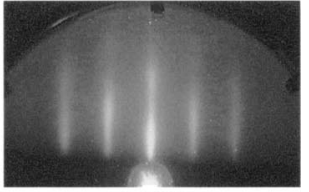

$\mathrm{MgO}[110]$

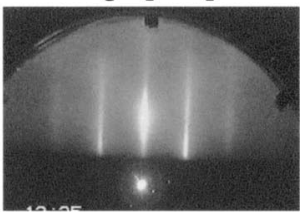

Fe [100]

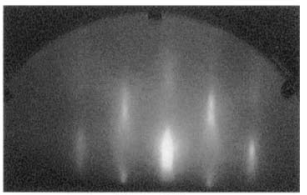

$\mathrm{Fe}[100]$
FIG. 2. RHEED patterns showing the epitaxy of the complete $\mathrm{Co} / \mathrm{Fe} / \mathrm{MgO} /$ $\mathrm{Fe}(001)$ heterostructure. (a) Faceted Fe deposited at RT on $\mathrm{MgO}(001)$, (b) Fe RHEED pattern after heat treatment at $400{ }^{\circ} \mathrm{C}$ during $10 \mathrm{~min}$, (c) $20 \AA$ $\mathrm{MgO}$ barrier, (d) $100 \AA$ Fe layer deposited at RT, (e) diffraction pattern corresponding to a deposit of $100 \AA \mathrm{Co}$ on the previous commented Fe layer at RT.

layers - was performed at RT without any annealing, to reduce as much as possible any interdiffusion between layers.

We now focus on the magnetic characterization by SQUID of the $\mathrm{Fe}(\mathrm{Co}) / \mathrm{MgO} / \mathrm{Fe}$ trilayers. As-grown square $5 \times 5 \mathrm{~mm}^{2}$ samples were measured in these experiments, previous to any lithographic process. In Fig. 3(a), the magnetic loops at 20 and $300 \mathrm{~K}$ for $\mathrm{Fe}_{0.5} \mathrm{Co}_{0.5}(200 \AA) / \mathrm{MgO}(20 \AA) / \mathrm{Fe}$ $(200 \AA)$ are shown with the magnetic field applied along the [100] Fe direction. Notice first that, at both temperatures, the magnetic electrodes are magnetically decoupled as shown by distinct and rather sharp magnetization transitions corresponding to independent magnetic switching of the electrodes. In Fig. 3(a), the magnitude of the transition is compared with the expected value for antiparallel alignment of the Fe bottom electrode and $\mathrm{Fe}_{0.5} \mathrm{Co}_{0.5}$ top electrode (dotted line) with the following assumptions: (1) the real thickness is the nominal one, (2) the lower coercive field transition corresponds to the bottom electrode magnetization switch, and (3) the magnetic moment for $\mathrm{Fe}$ and $\mathrm{Fe}_{0.5} \mathrm{Co}_{0.5}$ in the trilayer is the bulk value $\left(2.22 \mu_{B}\right.$ and $2.41 \mu_{B}$ per unit cell, respectively). The agreement between this estimate and the experimental results is very good, which makes the earlier assumptions plausible. Moreover, the coercive field of the bottom electrode, Fe $(200 \AA)$, in the trilayers at $300 \mathrm{~K}$ is about 10 
(a)

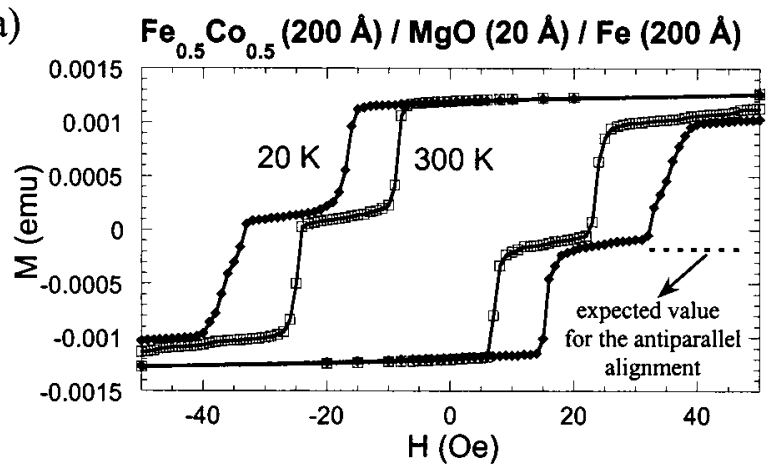

(b)

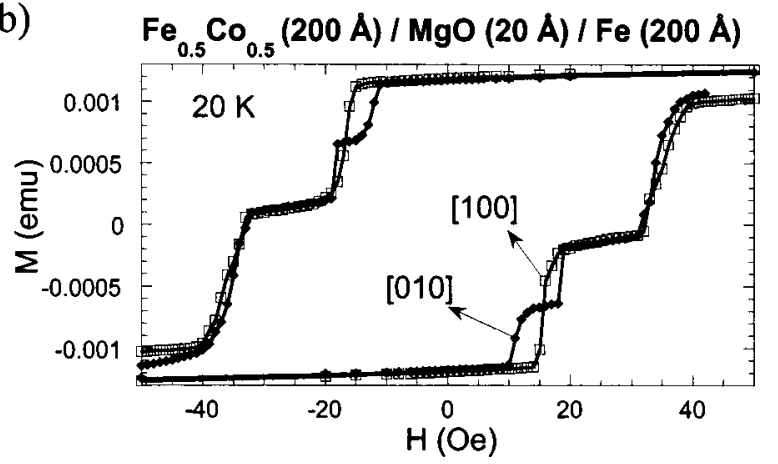

FIG. 3. SQUID magnetization loops for a $200 \AA \mathrm{FeCo} / 20 \AA \mathrm{MgO} / 200 \AA \mathrm{Fe}$ trilayer. (a) Temperature dependence of switching field at 20 and $300 \mathrm{~K}$. (b) Evidence of uniaxial in plane anisotropy of the Fe bottom electrode.

Oe, which coincides with the value observed by CostaKrämer et ll. $^{25}$ for single Fe $(200 \AA)$ thin films. The fact that the coercive field of the bottom electrode is not influenced by the growth of the barrier and the top electrode is a signature of the absence of substantial coupling between both electrodes in this trilayer. The coercive field of this Fe bottom electrode increases to about $16 \mathrm{Oe}$ at $20 \mathrm{~K}$, usually attributed to the decrease of the thermal energy that assists the magnetization reversal mechanism $\left(180^{\circ}\right.$ domain walls nucleation and propagation). In the case of the top electrode $\mathrm{Fe}_{0.5} \mathrm{Co}_{0.5}$ $(200 \AA$ ) the coercive field at $300 \mathrm{~K}$ is 24 Oe increasing to 35 Oe at $20 \mathrm{~K}$. It is worth it to bear in mind that the FeCo alloy for this composition displays a negative $K_{1}$ anisotropy constant, i.e., [110] crystallographic directions become now magnetically easy and [100] hard. ${ }^{26}$

In Fig. 3(b), a comparison of the magnetization loops applying the magnetic field along the [100] and [010] inplane crystallographic directions of the bottom $(\mathrm{Fe})$ electrode is shown. For the field applied along the [100] direction, two well-defined abrupt steps in the magnetization reversal are observed for each loop branch, corresponding to the reversal of the top and bottom electrode magnetizations. These reversals occur at moderate coercive fields of 16 and 35 Oe, respectively. On the other hand, on rotating the sample $90^{\circ}$ and applying the magnetic field along the other easy axis [010] Fe direction, an additional step at about 10 Oe is observed in the magnetization reversal process. This has been previously observed in single $\mathrm{Fe}(001)$ layers grown on $\mathrm{MgO}(001)$ substrates, ${ }^{25}$ and its origin has been attributed to an additional uniaxial magnetic anisotropy of interfacial origin and probably due to an in-plane distortion of the Fe lattice. This (a)

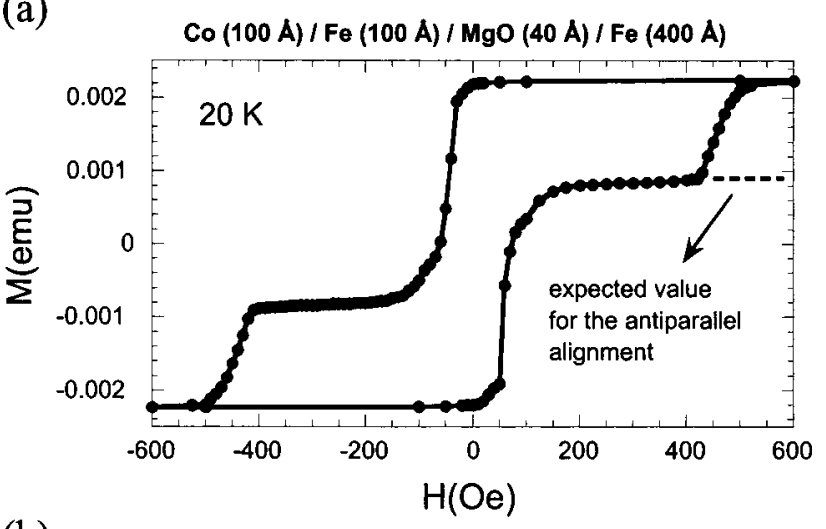

(b)

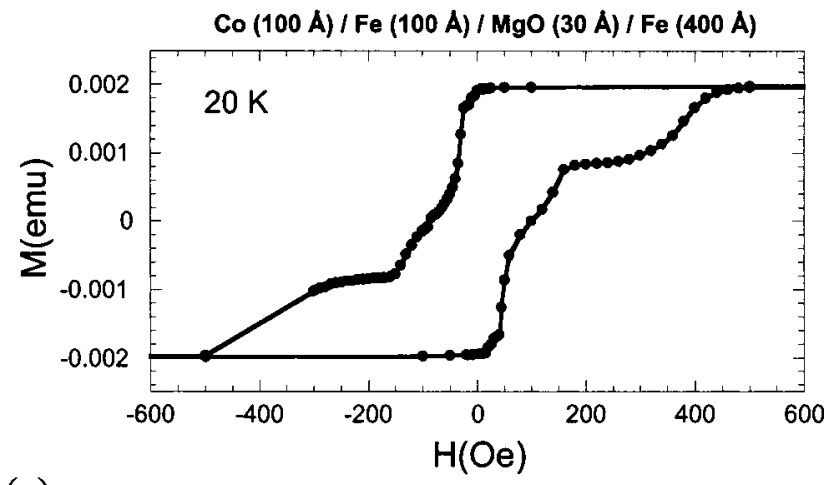

(c)

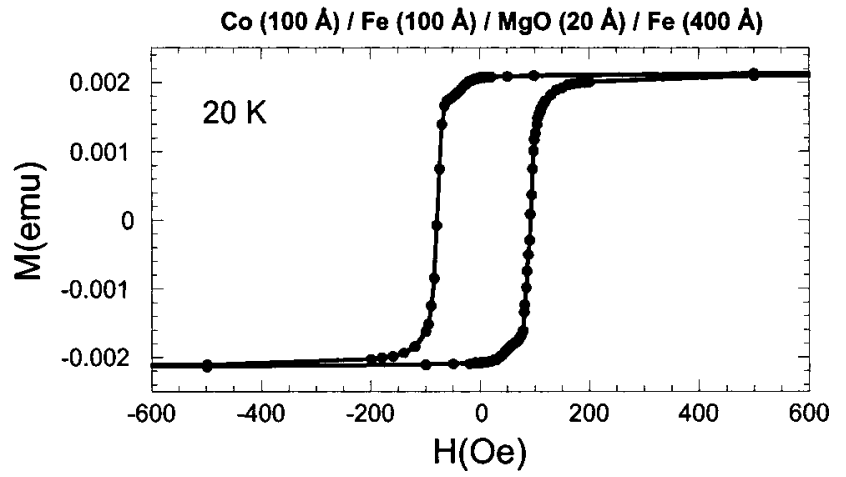

FIG. 4. SQUID magnetization loops at $20 \mathrm{~K}$ for $100 \AA \mathrm{Co} / 100 \AA \mathrm{Fe} / \mathrm{MgO}$ (d) $/ 400 \AA \mathrm{Fe}$ heterostructures with varying $\mathrm{MgO}$ barrier thicknesses (a) $d$ $=40 \AA$, (b) $d=30 \AA$, and (c) $d=20 \AA$. Magnetic field was applied along the $[100]$ Fe direction.

additional uniaxial anisotropy renders magnetically different directions that are cristallographically equivalent. This seems to be the case also for the $\mathrm{FeCo} / \mathrm{MgO} / \mathrm{Fe}$ trilayer. It is remarkable that for this high-quality crystalline heterostructure the magnetic anisotropy properties of the bottom electrode (closely related to its interfacial interaction with the substrate) still manifest after deposition of the complete trilayer structure without being affected by the deposition of uppermost layers. $^{27}$

Figure 4 shows the magnetic hysteresis loops at $20 \mathrm{~K}$ for three $100 \AA \mathrm{Co} / 100 \AA \mathrm{Fe} / \mathrm{MgO} / 400 \AA \mathrm{Fe}$ heterostructures where the composition and thickness of the top and bottom electrodes remain unchanged and the $\mathrm{MgO}$ thickness is varied from $40 \AA$ [Fig. 4(a)] to $30 \AA$ [Fig. 4(b)] and $20 \AA$ [Fig. 4(c)]. The hysteresis loop for the 40 - $\AA$-thick $\mathrm{MgO}$ spacer 
shows two clear magnetization reversals at 50 and $450 \mathrm{Oe}$ approximately. This indicates totally independent magnetic switching of top and bottom electrodes, with a large field range $(\sim 300 \mathrm{Oe})$ with an antiparallel magnetization configuration. Taking into account the bulk magnetic moments of $\mathrm{Fe}$ $\left(2.22 \mu_{B}\right)$ and Co $\left(1.72 \mu_{B}\right.$ per unit cell $)$ and using the nominal thickness of the electrodes, it is possible to assign the lower coercive field to the bottom electrode magnetization reversal and the higher coercive field to the top one [see Fig. 4(a)]. The measured high value of the coercive field of the top electrode, the bilayer $\mathrm{Co} / \mathrm{Fe}(450 \mathrm{Oe})$, shows that direct ferromagnetic coupling between $\mathrm{Co}$ and $\mathrm{Fe}$ is very effective increasing the coercive field of the combined electrode. This effect has also been reported for $\mathrm{Ni} / \mathrm{Fe}$ bilayers ${ }^{27}$ and by Faure-Vincent et al. for $\mathrm{Co} / \mathrm{Fe}$ on $\mathrm{MgO} / \mathrm{Fe}^{9}{ }^{9}$

The morphology of the individual electrodes was also characterized ex situ by AFM measurements for the different samples. In the case of the bottom Fe layer, a rms roughness of $3 \AA$ was obtained. For the case of the double Co/Fe layer structure, AFM inspection revealed an increased rms roughness of $8 \AA$. This coarseness arises from the defective Co deposition on top the faceted Fe layer, as shown in Figs. 2(d) and 2(e). Since orange-peel type of coupling between both electrodes would be ferromagnetic only for the case of roughness at the barrier/bottom electrode interface, which would produce correlated interface topography of both magnetic layers in the case of $\mathrm{MgO}$ barrier conformal growth, the orange-peel type of coupling in these heterostructures is expected to be negligible because of the sharp $\mathrm{Fe} / \mathrm{MgO} / \mathrm{Fe}$ interfaces. Additional evidence of atomically flat $\mathrm{Fe} / \mathrm{MgO} / \mathrm{Fe}$ interfaces comes by high-resolution TEM observations reported in Ref. 15.

For the thinnest $\mathrm{MgO}$ spacer $(20 \AA)$ the hysteresis loop is almost square [see Fig. 4(c)], with a single abrupt magnetization reversal. This is due to the simultaneous reversal of top and bottom layer magnetizations, probably due to the presence of pinholes in the $\mathrm{MgO}$ spacer. The differences between Figs. 3(a) and 4(c) for the same $\mathrm{MgO}$ barrier thickness could indicate in this last case some deviation from nominal deposited thicknesses.

In the case of the $30 \AA \mathrm{MgO}$ thickness [see Fig. 4(b)], the situation is intermediate between the 20 and $40 \AA$ cases. Magnetization reversal appears to proceed in three stages, with two clear transitions at 50 and $400 \mathrm{Oe}$, and a rotation stage at intermediate fields characteristic of coupled media. This is probably because pinholes present in the 20- $\AA$-thick $\mathrm{MgO}$ spacer sample shown in Fig. 4(c) have almost completely disappeared in the $30-\AA ̊$-thick one.

Up to now, results on epitaxial trilayer systems of macroscopic (millimeter) dimensions have been presented, where dipolar type interactions due to edge accumulation of poles do not play a relevant role. In what follows this study is extended to similar structures but of micrometer lateral dimensions defined by photo and e-beam lithography. Kerr magnetization loops for a nominal vertical structure of $100 \AA$ $\mathrm{Co} / 100 \AA \mathrm{Fe} / 30 \AA \mathrm{MgO} / 400 \AA \mathrm{Fe}$ are shown in Fig. 5 for elements of different shapes and sizes: circular elements of 18, 28, and $43 \mu \mathrm{m}$ diameter [Fig. 5(a)] and a square element of $700 \mu \mathrm{m}$ lateral dimensions [Fig. 5(b)]. All structures are

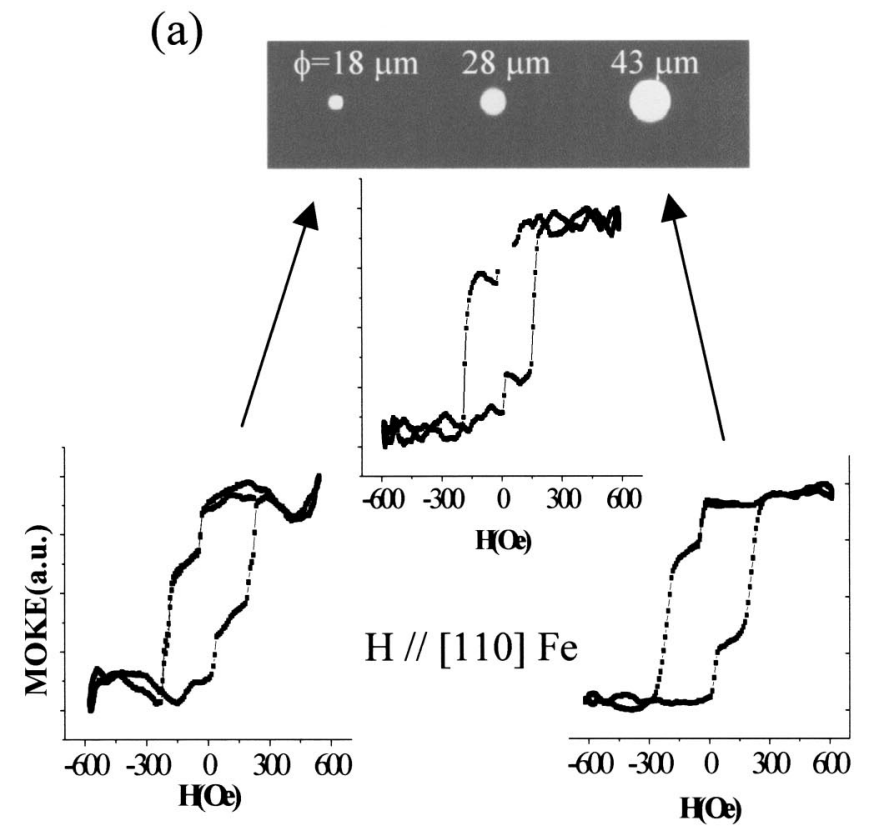

(b)

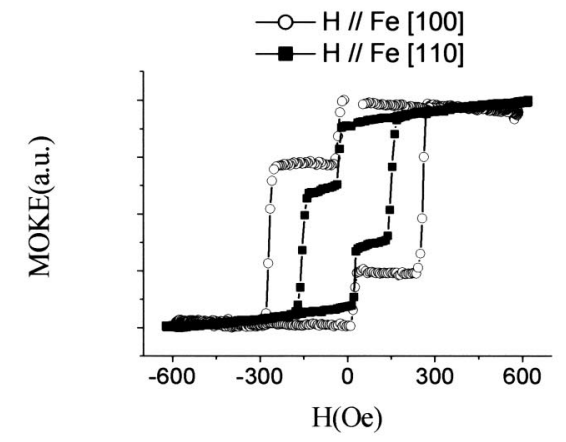

FIG. 5. MOKE hysteresis loops for a $100 \AA \mathrm{Co} / 100 \AA \mathrm{Fe} / 30 \AA \mathrm{MgO} / 400 \AA$ Fe structure patterned into elements of different sizes and shapes. (a) Circular elements of different sizes (magnetic field applied along [110] Fe direction), (b) squared single element of $700 \mu \mathrm{m}$ lateral dimension.

patterned on the same macroscopic sample, which guarantees identical deposition conditions and layer thicknesses. While these circular structures can clearly be differentiated from macroscopic samples, the square one has dimensions in the millimeter range. In Fig. 5(a) the hysteresis loops for each circular trilayer structure measured by focused MOKE at RT are shown. The magnetic field is applied along the [110] Fe hard axis. Even though the signal is noisy due to the small amount of probed material, two clear magnetization reversal steps are observed for the three junctions, demonstrating independent magnetization switching of top and bottom electrodes.

For the square shaped structure the Kerr hysteresis loops [Fig. 5(b)] measured with the magnetic field applied along the in plane [100] easy and [110] hard Fe directions clearly show the two sharp and independent magnetization reversals as well. These are similar to those observed for the macroscopic samples shown in Fig. 4(a). Despite of its still macroscopic size, the reduction in lateral dimensions produces a 
better definition of the magnetization reversal in this square structure. In the case of the circular elements, and due to their microscopic dimensions, an additional term due to dipolar coupling needs be taken into consideration to understand their magnetization reversal, ${ }^{12}$ nevertheless, no significant differences are noticeable after comparison of hysteresis loops of circular junctions with those for square junctions.

As pointed out before, for the thinnest $\mathrm{MgO}$ barriers the hysteresis loop is nearly square due to the simultaneous reversal of the top and bottom layer magnetizations, probably originated by the presence of pinholes in the $\mathrm{MgO}$ spacer. The role of pinholes on the magnetization reversal for junctions as a function of the lateral size, for a thin $10 \AA \mathrm{MgO}$ barrier, is further illustrated in Fig. 6. The film structure in this case is $100 \AA \mathrm{Fe} / 10 \AA \mathrm{MgO} / 100 \AA \mathrm{Fe}$, i.e., identical top and bottom electrodes. As observed in Fig. 6(a), the continuous macroscopic film displays a square loop with a coercive field of $10 \mathrm{Oe}$, demonstrating the simultaneous reversal of top and bottom electrode magnetizations like in a single $\mathrm{Fe}$ layer film. ${ }^{25}$ The sample is patterned by e-beam lithography and ion beam etching, as described in Ref. 12, and the resulting array of square junctions is measured by focusing a light spot, about $100 \mu \mathrm{m}$ diameter, within the square $250 \mu \mathrm{m}$ edge array. The measured magneto optical Kerr loop corresponds then to the behavior of several hundreds to thousands of microjunctions. Figure 6(b) shows selected hysteresis loops for arrays with different lateral sizes of the microjunctions, with the field applied along an easy $\mathrm{Fe}$ [100] direction. For junctions larger than $3 \mu \mathrm{m}$, the reduced remanence, $M_{R}=M / M_{S \mid H=0}$, of the array is close to one, pointing to top and bottom Fe electrodes that are exchange coupled. In other words, most of the array still behaves like the continuous film. However, for junctions smaller than $3 \mu \mathrm{m}$ the reduced remanence decreases, and loops evidence a reversal by two stages with a remanent state close to zero magnetization along the applied field direction. This indicates that the two electrodes magnetizations orient in an antiparallel fashion for a large fraction of the array elements, and is explained by the magnetostatic energy reduction obtained by the antiparallel orientation of the electrodes magnetizations. For this barrier thickness of $10 \AA \mathrm{MgO}$, the effect of patterning on the magnetic loops is different of what has been measured for 20,50, and $70 \AA \mathrm{MgO},{ }^{12}$ where the two stage magnetization reversal is measured for all lateral sizes. Furthermore, and differently from the case of single layer $(200 \AA \mathrm{Fe})$ tiles that break into domains for sizes smaller $3 \mu \mathrm{m},{ }^{25}$ a more stable single domain structure due to the flux closure between the top and bottom electrodes is achieved in each trilayered tile. ${ }^{12}$ All these experimental facts prove that pinholes in the barrier are the main cause of the observed behavior. In this scheme, the system behaves as if there was a threshold number of pinholes (or pinholes of a certain size) above which the two electrodes are coupled ferromagnetically [see a simplified illustration in Fig. 6(c)]. In this scheme, patterning the structure and decreasing the size of the elements decreases the number of junctions coupled via pinholes increasing the area occupied by exchange uncoupled junctions. (a)

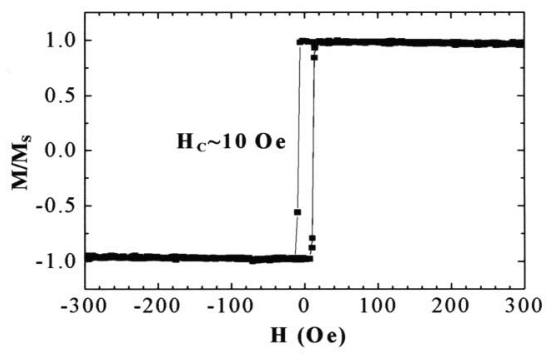

(b)

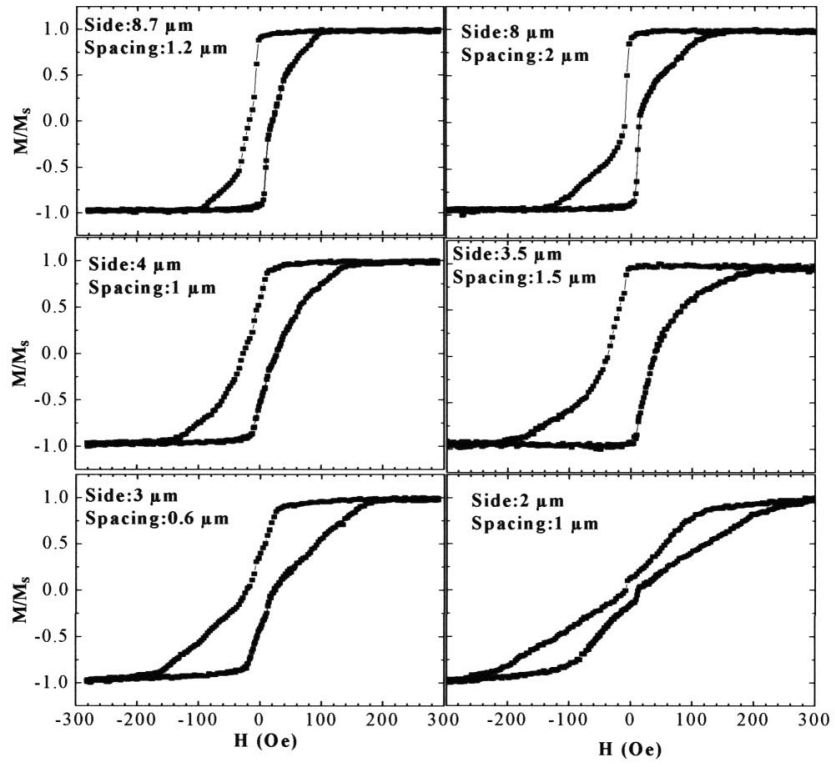

(c)
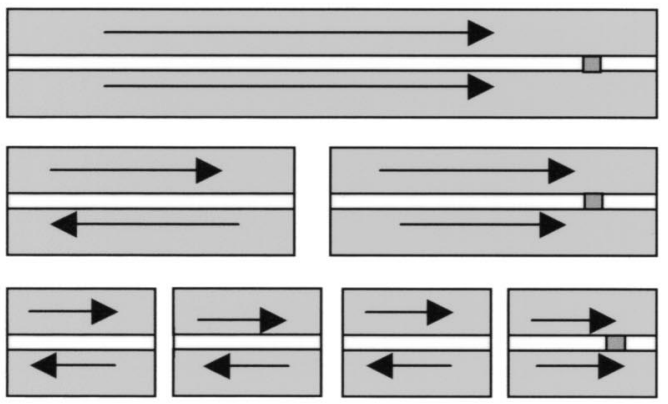

FIG. 6. MOKE hysteresis loops for a $100 \AA \mathrm{Fe} / 10 \AA \mathrm{MgO} / 100 \AA$ A Fe trilayer (field applied along the [100] Fe direction). (a) Continuous unpatterned film, (b) arrays of squared junctions with different lateral sizes ranging from 9 to $2 \mu \mathrm{m}$, and different interelement separations. (c) simplified scheme of the effect patterning has on the coupling mediated by pinholes as a function of the trilayer lateral size. The percentage of tiles with pinholes is reduced from $100 \%$ ( 1 out of 1 ) for the first case, $50 \%$ ( 1 out of 2 ) in the second case, and $25 \%$ ( 1 out of 4 ) in the third one.

\section{CONCLUSIONS}

Multilayered $\mathrm{TM} / \mathrm{MgO} / \mathrm{Fe}(001)$ heterostructures (TM: $\mathrm{FeCo}, \mathrm{Co} / \mathrm{Fe}$, and $\mathrm{Fe}$ ) are grown epitaxially and the magnetic coupling between top and bottom electrodes is studied changing the insulating $\mathrm{MgO}$ barrier thickness and the lateral dimensions of the structures. The crystal quality is investigated by RHEED in situ at different stages of the growth of 
the $\mathrm{TM} / \mathrm{MgO} / \mathrm{Fe}(001)$ heterostructures. Magnetic characterization by SQUID (macroscopic structures) and transverse Kerr effect (microscopic structures) shows clear independent switching of top and bottom electrodes at large (40 ̊) spacer thickness. Remarkably, the bottom Fe electrode anisotropies are unaffected by the subsequent layers depositions that complete the heterostructure. The magnetic behavior on reducing the $\mathrm{MgO}$ epitaxial insulating barrier thickness indicates the presence of pinholes for the thinnest spacers. This gives rise to ferromagnetic coupling due to direct contact between both electrodes. However, for very thin barriers, patterning the structure and decreasing the lateral size of the elements decreases the percentage of junctions coupled via pinholes.

\section{ACKNOWLEDGMENTS}

The Spanish Commission of Science and Technology, and Comunidad de Madrid are acknowledged for financial support.

${ }^{1}$ J. S. Moodera, L. R. Kinder, T. M. Wong, and R. Meservey, Phys. Rev. Lett. 74, 3273 (1995).

${ }^{2}$ S. S. P. Parkin et al., J. Appl. Phys. 85, 5828 (1999).

${ }^{3}$ W. H. Butler, X. G. Zhang, T. C. Schultess, and J. M. MacLaren, Phys. Rev. B 63, 54416 (2001).

${ }^{4}$ J. F. Bobo, H. Kikuchi, O. Redon, E. Snoeck, M. Piecuch, and R. L. White, Phys. Rev. B 60, 4131 (1999).

${ }^{5}$ D. J. Keavney, E. E. Fullerton, and S. D. Bader, J. Appl. Phys. 81, 795 (1997).

${ }^{6}$ P. A. A. van der Heijden, P. J. H. Bloemen, J. M. Metselaar, R. M. Wolf,
J. M. Gaines, J. T. W. M. van Eemeren, P. J. van der Zaag, and W. J. M. de Jonge, Phys. Rev. B 55, 11569 (1997).

${ }^{7}$ L. Néel, C. R. Hebd. Seances Acad. Sci. 255, 1676 (1962).

${ }^{8}$ E. Popova et al., Appl. Phys. Lett. 81, 1035 (2002).

${ }^{9}$ J. Faure-Vincent J, C. Tiusan, C. Bellouard, E. Popova, M. Hehn, F. Montaigne, and A. Schuhl, Phys. Rev. Lett. 89, 107206 (2002).

${ }^{10}$ J. C. Slonczewski, Phys. Rev. B 39, 6995 (1989).

${ }^{11}$ P. Bruno, Phys. Rev. B 52, 411 (1995).

${ }^{12}$ J. L. Costa-Krämer, J. Anguita, J. I. Martín, C. Martínez-Boubeta, A. Cebollada, and F. Briones, Nanotechnology 13, 695 (2002).

${ }^{13}$ M. Bowen et al., Appl. Phys. Lett. 79, 1655 (2001).

${ }^{14}$ E. Popova et al., Appl. Phys. Lett. 81, 1035 (2002).

${ }^{15}$ C. Martínez Boubeta, E. Navarro, A. Cebollada, F. Briones, F. Peiró, and A. Cornet, J. Cryst. Growth 226, 223 (2001).

${ }^{16}$ W. H. Lee et al., Appl. Phys. Lett. 77, 2192 (2000).

${ }^{17}$ P. Liu, T. Kendelewicz, J. G. E. Brown, and G. A. Parks, Surf. Sci. 412413, 287 (1998).

${ }^{18}$ J. Palomares, C. Martínez Boubeta, and A. Cebollada (unpublished).

${ }^{19}$ M. Hansen, Constitution of Binary Alloys (McGraw Hill, New York, 1958).

${ }^{20}$ W. C. Ellis and E. S. Greiner, Trans. Am. Soc. Met. 29, 415 (1941).

${ }^{21}$ K. Thürmer, R. Koch, M. Weber, and K. H. Rieder, Phys. Rev. Lett. 75, 1767 (1995).

${ }^{22}$ J. Zhang, Z. L. Han, S. Varma, and B. P. Tonner, Surf. Sci. 298, 351 (1993).

${ }^{23}$ G. C. Gazzadi and S. Valeri, Europhys. Lett. 45, 501 (1999).

${ }^{24}$ S. K. Kim, C. Petersen, F. Jona, and P. M. Marcus, Phys. Rev. B 54, 2184 (1996).

${ }^{25}$ J. L. Costa-Krämer, J. L. Menéndez, A. Cebollada, F. Briones, D. García, and A. Hernando, J. Magn. Magn. Mater. 210/1-3, 341 (2000).

${ }^{26}$ Soshin Chikazumi, Physics of Ferromagnetism (Oxford University Press, New York, 1997).

${ }^{27}$ M. Przybylski, J. Grabowski, F. Zavaliche, W. Wulfhekel, R. Scholz, and J. Kirschner, J. Phys. D 35, 1821 (2002). 